\title{
PLANAR LINE FAMILIES. I
}

\section{P. C. HAMMER AND ANDREW SOBCZYK}

Introduction. In this paper we introduce a definition of outwardly simple line families, suggested by the work of one of the authors [1], which associated certain families of extended diameters with planar convex bodies. While we have made the obvious extensions of this definition to $n$-dimensional space, in this paper we are concerned only with planar line families.

A family $F$ of lines in the affine plane is said to be outwardly simple if it covers every point (including points at infinity) exterior to some circle simply. In this paper we develop some properties of outwardly simple line families, showing that all such may be obtained from the class of continuous increasing functions mapping the closed interval $[0,1]$ into itself. We then show that each outwardly simple line family is the set of extended diameters of a constant-breadth closed convex curve. Finally, it is shown how to construct all constantbreadth convex curves in the plane.

The first known work on the construction of constant-breadth convex curves in the plane is due to Euler [2]. See [3, pp. 47-51] and [4, pp. 130-139] for discussion and references. Our work completes the earlier work in giving essentially a method of constructing all constant-breadth convex curves in the plane.

Outwardly simple line families. Let $F$ be an outwardly simple line family and let $\beta$ be a circle containing all intersection points of pairs of lines in $F$ in its interior. Then $\beta$ is simply covered by lines in $F$ and every pair of lines in $F$ intersects in the interior of $\beta$. Let a line $m$ in $F$ intersect $\beta$ in the points $x$ and $y$. Define a transformation $T$ of $\beta$ into itself by $T(x)=y, T(y)=x$.

THEOREM 1: The transformation $T$ is a continuous involutory transformation without fixed point of $\beta$ into itself.

PROof. That $T$ is involutory and has no fixed point is clear. That $T$ is continuous follows, since each point and image pair $x_{1}, T\left(x_{1}\right)$ separates any other point and image pair $x_{2}, T\left(x_{2}\right)$.

Corollary to Theorem 1. Let $\gamma$ be a simple closed curve and let $T$ be an involutory transformation of $\gamma$ into itself with the property that each point and image pair separates every other such pair on $\gamma$. Then $T$

Presented to the Society, April 27, 1951; received by the editors May 7, 1951 and, in revised form, June 20, 1952. 
is continuous and has no fixed point.

Now, let a cartesian coordinate system $(u, v)$, with origin at the center of $\beta$, say, be chosen. Then the family of $F$ lines can be written in the form

$$
u \sin \alpha-v \cos \alpha-p(\alpha)=0, \quad 0 \leqq \alpha<\pi .
$$

The continuity of $p(\alpha)$ follows from the continuity of $T$. Now when $\alpha \nearrow \pi$, continuity requires that $p(\alpha) \rightarrow-p(0)$ since the directions $\alpha=0$ and $\alpha=\pi$ coincide. For convenience we extend the range of $p(\alpha)$ to the closed interval $[0, \pi]$ and write $p(0)=-p(\pi)$.

THEOREM 2. An outwardly simple line family covers every point in the plane.

Proof. Let $\left(u_{0}, v_{0}\right)$ be any point in the plane and consider the continuous function of $\alpha$ :

$$
f(\alpha)=u_{0} \sin \alpha-v_{0} \cos \alpha-p(\alpha) .
$$

Now $f(0)=-v_{0}-p(0)$ and $f(\pi)=v_{0}-p(\pi)$. Since $p(0)=-p(\pi), f(0)$ $=-f(\pi)$ and hence there is an angle $\alpha_{0}, 0 \leqq \alpha_{0}<\pi$, such that $f\left(\alpha_{0}\right)=0$. Hence $\left(u_{0}, v_{0}\right)$ is on the line in $F$ with direction angle $\alpha_{0}$. Q.E.D.

Since the outwardly simple line family $F$ covers the entire plane, there is a line in $F$ through the center of the circle $\beta$. Let the $u$-axis be taken along this line with origin at the center. Let $x(\alpha)$ and $y(\alpha)$ be points on $\beta$ which are on the line with direction angle $\alpha$ in $F$ and let $x(\alpha)$ be in the half-plane $v>0$ and $y(\alpha)$ in the half-plane $v<0$. Let the angle of the circular arc from the positive $u$-axis to $x(\alpha)$ be $\theta_{1}$ and the angle of the arc from the negative $u$-axis to $y(\alpha)$ be $\theta_{2}$. Then $\theta_{2}$ is an increasing continuous function of $\theta_{1}, \theta_{2}=g\left(\theta_{1}\right)$ such that $g(0)=0, g(\pi)=\pi$.

It is readily seen that if any continuous increasing function $g\left(\theta_{1}\right)$ maps $[0, \pi]$ into $[0, \pi]$, we can determine an outwardly simple line family from it.

THEOREM 3. Every outwardly simple line family in the plane determines a continuous increasing function which maps $[0, \pi]$ into itself, and conversely.

We mention a few results which may be readily established. Every continuous involutory transformation of a circle into itself without fixed point generates a continuous increasing function mapping $[0,1]$ into itself and conversely. Every such involutory transformation determines an outwardly simple line family and conversely. 
Given a continuous involutory transformation, without fixed point, of a circle into itself, then the line segments joining point and image pairs cover the circular disk. This last result, generalized suitably, has been established for arbitrary $n$ in [5].

Extended diameters of convex bodies. It is shown in [1] that the extended diameters of a planar convex body cover the plane. It is further shown that one may pick a unique subfamily of diameters which extend to give an outwardly simple line family and which one is justified in calling the essential diameter family of the convex body. The question naturally arises: For a given outwardly simple line family $F$ does there exist a convex body with its essential diameters lying on lines in $F$ ? The question may be answered in the affirmative trivially if one omits the requirement "essential." For, let $m_{1}$ and $m_{2}$ be two perpendicular lines in $F$, let $\beta$ be a circle containing in its interior the intersection points of lines in $F$, and form a square containing $\beta$ with diagonals along $m_{1}$ and $m_{2}$. Then, every line in $F$ cuts the square in a diameter of the square. However, the essential diameters of a square all pass through its center.

Since we shall be dealing with constant-diameter-length convex bodies, we may review a few facts concerning them. First, if all the diameters of a convex body have the same length, then the distance between pairs of parallel contact lines of the body is constant. The diameters are perpendicular to their corresponding pairs of parallel contact lines. The diameters of a constant-diameter convex body extend to give an outwardly simple family of lines and hence all its diameters are essential.

TheOREM 4. Let $F$ be a given outwardly simple line family. Then there exists a convex body $C$ of constant diameter length such that its diameters extend to give $F$. Moreover, the boundary of $C$ may be taken as a smooth curve.

Proof. Let $\beta$ be a circle containing every intersection point of lines of $F$ in its interior. Let $m_{0}$ be any line in $F$. We designate one ray of $m_{0}$ with origin interior to $\beta$ by $m_{0}^{+}$. The complementary ray we designate by $m_{0}^{-}$. We use $m_{0}^{+}$as our zero angle indicator and measure all angles of rays or lines in the counterclockwise sense from it. Let $m$ be any line in $F$ not coinciding with $m_{0}$. Then the angle $\alpha$ of the line is the angle from $m_{0}^{+}$to the line, $0 \leqq \alpha<\pi$. Again we consider $m$ as divided into two rays with common origin inside $\beta$. The ray of $m$ making the angle $\alpha$ with $m_{0}^{+}$we term $m^{+}$and the complementary ray making an angle $\alpha+\pi$ we term $m^{-}$. 
Now choose the three lines of $F$ making angles $\pi / 4, \pi / 2$, and $3 \pi / 4$ with $m_{0}^{+}$. Label these lines $m_{1}, m_{2}, m_{3}$ respectively (see Fig. 1). Now we choose a point $a_{0}$ on $m_{0}^{+}$outside $\beta$ and drop a perpendicular from it to $m_{1}^{+}$. The foot of this perpendicular on $m_{1}^{+}$we call $p_{1}$. From $p_{1}$ drop a perpendicular to $m_{2}^{+}$and call the foot of this perpendicular $p_{2}$. We continue in this fashion dropping perpendiculars successively to $m_{1}^{+}, m_{2}^{+}, m_{3}^{+}, m_{0}^{-}, m_{1}^{-}, m_{2}^{-}, m_{3}^{-}$, and $m_{0}^{-}$. Now, this process may be carried out provided no one of the perpendiculars enters the circle $\beta$. However, it is readily seen that if $a_{0}$ is chosen far enough

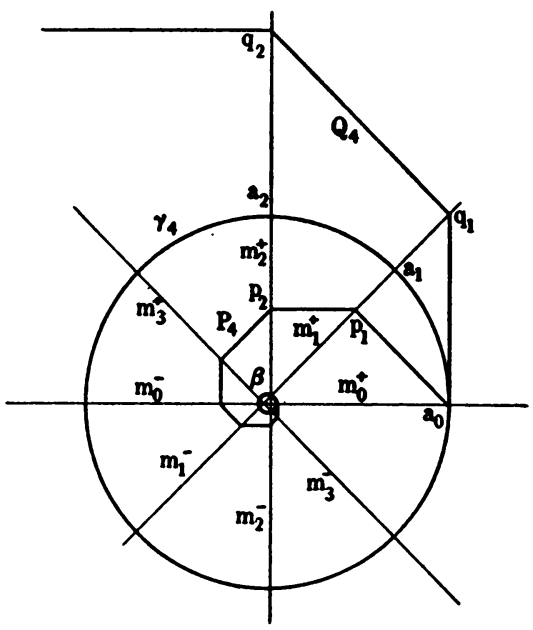

FIG. 1

from $\beta$ on $m_{0}^{+}$, the polygonal spiral, $P_{4}$, will be exterior to $\beta$. We assume $a_{0}$ to be so chosen. We shall later show that the terminal point $p_{8}$ of $P_{4}$ is on $m_{0}^{+}$between $\beta$ and $a_{0}$.

Now, starting again at $a_{0}$ on $m_{0}^{+}$, erect a perpendicular to $m_{0}^{+}$and extend until it intersects $m_{1}^{+}$and $q_{1}$. At $q_{1}$ on $m_{1}^{+}$erect a perpendicular intersecting $m_{2}^{+}$at $q_{2}$ and so on until we have "encircled" $\beta$ and returned to $m_{0}^{+}$at a point $q_{8}$ which will be seen to be outside $a_{0}$ on $m_{0}^{+}$. This polygonal spiral we designate by $Q_{4}$.

Next, let $x_{i j}$ be the intersection point of $m_{i}$ and $m_{j}$ where $i \neq j$. Now with $x_{01}$ as center and the length of $x_{01} a_{0}$ as radius sweep a circular arc from $a_{0}$ on $m_{0}^{+}$to $a_{1}$ on $m_{1}^{+}$. Then with $x_{12}$ as center and the length of $x_{12} a_{1}$ as radius describe a circular arc from $a_{1}$ to $a_{2}$ on $m_{2}^{+}$. Continuing in this manner we obtain 8 circular arcs and it is seen that these arcs form a closed convex curve of constant diameter length. This curve we designate as $\boldsymbol{\gamma}_{4}$. The convexity and constancy 
of diameter of $\gamma_{4}$ follow since the curve is locally convex everywhere and since the sum of "opposite" radii of arcs is constant. Furthermore, $\gamma_{4}$ separates $P_{4}$ and $Q_{4}$ except at $a_{0}$ where all these curves have a point in common. Hence $p_{8} q_{8}$ on $m_{0}^{+}$contains $a_{0}$ in its interior.

The next step, clearly, is to refine the angular increments between lines. To do this we use the same lines as previously and take the next four from $F$ so that the angular increment is $\pi / 8$. Then we generate polygonal spirals $P_{8}$ and $Q_{8}$ and a closed convex curve $\gamma_{8}$ of constant diameter length exactly as before. We observe that $P_{8}$ has only one point, $a_{0}$, in common with $P_{4}$ and that it is further from the circle $\beta$ on every ray of $F$ than $P_{4}$ except at $a_{0}$. Furthermore, $Q_{8}$ has its first line segment along the first of $Q_{4}$ but otherwise $Q_{8}$ is "inside" of $Q_{4}$. As before, $\gamma_{8}$ separates $P_{8}$ and $Q_{8}$ except for the common point $a_{0}$.

The steps in an inductive procedure for determining a sequence of curves $p_{n}, Q_{n}$, and $\gamma_{n}, n=4 \cdot 2^{k}, k=0,1,2, \cdots$, are now clear. Letting $n$ be fixed and ignoring the previous designation of points on the curves we let $a_{0}, p_{1}, \cdots, p_{2 n}$ be "corner" points of $P_{n} ; a_{0}, q_{1}, \cdots$, $q_{n}$ the arcs of $\gamma_{n}$. Let the successive radii of circular arcs of $\gamma_{n}$ be $r_{1}, r_{2}, \cdots, r_{2 n}$. The length of $p_{i} a_{i}$ is then exactly

$$
(1-\cos \alpha)\left[r_{i}+r_{i-1} \cos \alpha+r_{k-2} \cos ^{2} \alpha+\cdots+r_{1}(\cos \alpha)^{i-1}\right]
$$

and the length of $a_{i} q_{i}$ is

$$
(\sec \alpha-1)\left[r_{i}+r_{i-1} \sec \alpha+\cdots+(\sec \alpha)^{i-1}\right]
$$

where $\alpha=\pi / n$. Now, the $r_{i}$ are uniformly bounded and $(\sec \alpha)^{2 n}$ approaches unity as $n$ increases. Hence, the length $p_{i} q_{i}=p_{i} a_{i}+a_{i} q_{i}$ goes uniformly to zero since $1-\cos \alpha$ and $\sec \alpha-1$ both vanish with the order $1 / n^{2}$ and the sums of terms in parentheses are of order $n$ in both cases. Now since $\left\{P_{n}\right\}$ is an outwardly moving sequence of curves along rays of lines in $F$ and $\left\{Q_{n}\right\}$ is an inwardly moving sequence of curves, they have a common limit curve which is also the limit curve of $\left\{\gamma_{n}\right\}$. This limit curve we call $\boldsymbol{\gamma}$. It is a convex curve bounding a constant-diameter-length convex body $C$. However, we want to show that the boundary of $\boldsymbol{\gamma}$ is smooth, i.e., that at every point of $\boldsymbol{\gamma}$ there is a unique tangent line.

We change our mode of procedure. Suppose that $m_{0}^{+}$is the positive $v$-axis in a cartesian coordinate system and that the positive $u$-axis is along $m_{1}^{+}$where $m_{1}$ is the line in $F$ perpendicular to $m_{0}$. Consider the differential equation

$$
\frac{d v}{d u}=-\tan \alpha
$$


subject to the initial condition $\left(u=0, v=v_{0}\right)$ where $\left(0, v_{0}\right)$ is the point $a_{0}$. This is the differential equation of normals to the family $F$. Now it may be established in an appreciable open region containing the point $a_{0}$ that the Lipschitz condition is satisfied and hence the existence of a unique solution arc through $a_{0}$ is assured. It can also be established that the part of this arc starting at $a_{0}$ and proceeding for increasing $\alpha$ 's lies between $P_{n}$ and $Q_{n}$ along rays of $F$ for every $n$. Hence this arc lies on $\gamma$. One may then change the axis designation and extend the arc in a finite number of steps completely around $\beta$. Since these solution arcs all lie on $\gamma$, the curve finally closes at $a_{0}$. Hence every point of $\boldsymbol{\gamma}$ has a unique tangent line and the directions of these lines change continuously. Furthermore, the tangent lines are at right angles to lines of $F$ and hence $F$ is the extended diameter family of $C$. Moreover, the uniqueness of solution of the differential equation shows that $\gamma$ is the only curve cutting lines of $F$ normally and passing through $a_{0}$ or any other point on $\gamma$. It readily follows that if $a$ is a point on $m_{0}^{+}$further out than $a_{0}$, there is a unique curve $\gamma_{a}$ which is of constant diameter, convex, and which cuts lines of $F$ normally. The increase in the constant diameter length from $\gamma$ to $\gamma_{a}$ is twice the length $a_{0} a$. This can be seen from the polygonal spiral $P_{n}$. If we had started at $a$ instead of $a_{0}$ and constructed $P_{n}^{\prime}$, say, then the distance between corresponding points of $P_{n}$ and $P_{n}^{\prime}$ is $a a_{0}(\cos \pi / n)^{k}, k=0,1, \cdots, 2 n$. Since $(\cos \pi / n)^{k} \rightarrow 1$ as $n \rightarrow \infty$, we have that $\gamma_{a}$ has a diameter increase over $\gamma$ by twice $a_{0} a$. The convex body of Theorem 4 is the body bounded by $\gamma$. Q.E.D.

Construction of all planar convex bodies of constant diameter length. We have shown how to construct all outwardly simple families of lines in the plane, given the set of all increasing continuous functions mapping $[0,1]$ into $[0,1]$. We shall now show how to determine the complete class of convex bodies of constant diameter length with diameters given along any outwardly simple family of lines. In order to do this, we review some results contained in [1].

Let $C$ be a closed planar convex body. If $C$ has a center point, then we say $C$ is completely reducible and in that case the essential diameters of $C$ all pass through the center. If $C$ has no center point, then there exists a unique convex sub-body $C_{i}$ of $C$ which has the property that its essential diameters extended by a constant ratio no less than one about their midpoints are precisely the essential diameters of $C$. Furthermore, $C_{i}$ is the smallest sub-body of $C$ for which this is true. In particular, $C_{i}$ may be $C$ in which case we say $C$ is irreducible. Otherwise we say $C$ is reducible to $C_{i}$. Every set obtained by extending the essential diameters of $C_{i}$ about their midpoints 
with a fixed ratio greater than unity is a closed convex body reducible to $C_{i} . C_{i}$ has the property that either at least one pair of its essential diameters intersects on the boundary or a boundary point of $C_{i}$ is a limit point of intersection points of essential diameters.

In view of these results, consider an outwardly simple family of lines, $F$, and the constant diameter convex body $C$ shown to exist in the proof of Theorem 4. If all the lines of $F$ are copunctal, $C$ is obviously a circle and the family of all the circles concentric with $C$ is the set of all constant diameter convex bodies with $F$ as the family of extended diameters. Now suppose that the lines of $F$ are not all copunctal. The constant diameter convex body $C$ has associated with it a unique irreducible convex sub-body $C_{i}$ which, in view of the above results, has a constant diameter length and the diameters of $C_{i}$ lie along the diameters of $C$, i.e., along the lines of $F$. One cannot shrink the diameters of $C_{i}$ about their midpoints with a fixed ratio to get a smaller convex body of constant diameter with diameters along $F$, since diameters of $C_{i}$ intersect arbitrarily close to the boundary or on the boundary of $C_{i}$. All convex bodies obtained by expanding the diameters of $C_{i}$ about their midpoints with a constant ratio give convex bodies of constant diameter length with diameters lying on the lines of $F$. These bodies we call the exterior associated bodies of $C_{i}$.

Suppose there were a convex body $B$ of constant diameter with diameters lying along lines of $F$ which is not $C_{i}$ or any of its exterior associates. Extend the diameters of $B$ by a constant ratio greater than 1 to $B^{\prime}$ taking the ratio so large that the boundary of $B^{\prime}$ is outside $C$. $B^{\prime}$ is a convex body of constant diameter. However, since the boundaries of the exterior associated bodies of $C_{i}$ simply cover the plane exterior to $C_{i}$, the boundary $\gamma^{\prime}$ of $B^{\prime}$ must have a point in common with one. Then by the uniqueness of constant diameter convex curves having $F$ as the family of extended diameters in that region, $\boldsymbol{\gamma}^{\prime}$ must be the boundary of one of the exterior associated bodies of $C_{i}$ and hence $B$ is either $C_{i}$ or one of its exterior associates, since $B^{\prime}$ is reducible to $C_{i}$.

THEOREM 5. If $F$ is an outwardly simple family of lines not all copunctal, then there exists a unique minimal convex body $C$ of constant diameter with diameters extending to give the family $F$. All the convex bodies of constant diameter with diameters lying along lines of $F$ are included in the set of bodies containing $C$ and its exterior associated bodies. Furthermore, all the exterior associated bodies of $C$ have smooth boundaries. $C$ itself may have angle points. 
Concluding remarks. We have exhibited, granted the family of all continuous increasing functions mapping $[0,1]$ onto itself, all outwardly simple line families in the plane, and all constant diameter convex bodies are orthogonal trajectories of these. The sense in which constant diameter convex bodies in the plane are generalizations of the circle is thus made clearer-the outwardly simple line families being a natural extension of pencils of lines.

In Planar line families. II, we shall develop the analytic characterization of outwardly simple line families and discuss the character of the intersection points of such families. P. C. Hammer has constructed an example of an outwardly simple line family in 3-space which cannot be obtained by extending the diameters of any convex body.

Several problems are suggested by the work in this paper. To mention one, what is the relationship among all increasing functions determined by a given outwardly simple line family in the manner described herein?

\section{REFERENCES}

1. P. C. Hammer, Convex bodies associated with a convex body, Proceedings of the American Mathematical Society vol. 2 (1951) pp. 781-793.

2. L. Euler, De Curvis Triangularibus, Acta Acad. Petropol vol. 2 (1778) pp. 3-30.

3. D. J. Struik, Differential geometry, Cambridge, 1950.

4. T. Bonnesen and W. Fenchel, Konvexe Koerper, Ergebnisse der Mathematik, Berlin, 1934.

5. Amasa Forrester, $A$ theorem on involutory transformations without fixed points, Proceedings of the American Mathematical Society vol. 3 (1952) pp. 333-334.

Los Alamos Scientific Laboratory 